Research Paper

\title{
Frizzled7 Promotes Epithelial-to-mesenchymal Transition and Stemness Via Activating Canonical Wnt/ $\beta$-catenin Pathway in Gastric Cancer
}

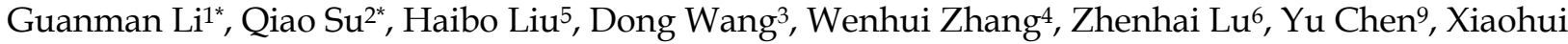 \\ Huang1, Wen Li1 ${ }^{1}$, Changhua Zhang77, Yulong $\mathrm{He}^{7}$, Li Fu' ${ }^{8}$, Jiong Bi ${ }^{\circledR}$
}

1. Laboratory of General Surgery, The First Affiliated Hospital, Sun Yat-Sen University, Guangzhou 510080, Guangdong, China

2. Animal Center, The First Affiliated Hospital, Sun Yat-Sen University, Guangzhou 510080, Guangdong, China

3. Department of Clinical Laboratory, The First Affiliated Hospital, Sun Yat-Sen University, Guangzhou 510080, Guangdong, China

4. Department of Pathology, The First Affiliated Hospital, Sun Yat-Sen University, Guangzhou 510080, Guangdong, China

5. Key Laboratory for Major Obstetric Diseases of Guangdong Province, The Third Affiliated Hospital of Guangzhou Medical University, Guangzhou Medical University, Guangzhou 510000, Guangdong, China

6. Department of Colorectal Surgery, Sun Yat-sen University Cancer Center, Guangzhou 510060, Guangdong, China

7. Gastrointestinal Surgery Center, The First Affiliated Hospital, Sun Yat-sen University, Guangzhou 510080, Guangdong, China

8. Guangdong Key Laboratory for Genome Stability \& Disease Prevention, Department of Pharmacology and Cancer Research Centre, School of Medicine, Shenzhen University, Shenzhen, China

9. Department of Immunity, Zhongshan School of Medicine, Sun Yat-Sen University, Guangzhou 510080, Guangdong, China

* These authors contributed equally to the study.

$\square$ Corresponding author: Jiong Bi, Laboratory of General Surgery, The First Affiliated Hospital, Sun Yat-Sen University, No.58 Zhongshan Rd.2, Guangzhou 510080, Guangdong, China. Tel/Fax: 86-20-87769673, E-mail: bijiong@mail.sysu.edu.cn

(c) Ivyspring International Publisher. This is an open access article distributed under the terms of the Creative Commons Attribution (CC BY-NC) license (https://creativecommons.org/licenses/by-nc/4.0/). See http://ivyspring.com/terms for full terms and conditions.

Received: 2017.11.09; Accepted: 2018.01.06; Published: 2018.02.12

\begin{abstract}
Aberrant activation of Wnt signaling is a crucial event in tumor development and metastasis. Wnt signaling is commonly divided into canonical and non-canonical signaling pathways based on whether $\beta$-catenin is activated (canonical). The two signaling pathways are initiated by Wnt ligand binding to the surface Frizzled (FZD) receptors, and regulate cancer stem cell self-renewal and epithelial-mesenchymal transition (EMT). Frizzled 7 (FZD7), a member of Frizzled family, promotes cell proliferation and invasiveness in many cancers, suggesting that FZD7 transmitting Wnt signaling is important for driving cancer growth. FZD7 expression has been reported to be up-regulated in human primary gastric cancer tissues. However, the molecular mechanism by which FZD7 promotes gastric cancer(GC) development and progression is not fully understood. Our present study showed that FZD7 was overexpressed in clinical GC samples, and thus was correlated with tumor invasion, lymphatic and organ metastasis, late TNM stages and poor patient survival. The endogenous expression of FZD7 was significantly increased in cancer stem cell-enriched spheres compared with adherent cells. Furthermore, RNA interference-mediated silencing of FZD7 inhibited proliferation, migration and invasion in gastric cancer cells. Moreover, ablation of FZD7 down-regulated EMT and the expression levels of cancer stem cell markers, and these inhibitions were associated with attenuated canonical $\mathrm{Wnt} / \beta$-catenin signaling. The results suggest that $\mathrm{Wnt}$ canonical pathway may contribute to tumorigenesis and metastasis, indicating that FZD7 could be a potential therapeutic target for gastric cancer.
\end{abstract}

Key words: FZD7; gastric cancer; cancer stem cell; epithelial-mesenchymal transition; Wnt/ $\beta$-catenin pathway

\section{Introduction}

Gastric cancer is one of the most common lethal malignancies worldwide. It was estimated that
951,600 gastric cancer cases and 723,100 deaths occurred in 2012 [1]. More importantly, above 50\% 
gastric cancers were diagnosed in China. Although early detection has substantially improved the prognosis of patients, gastric cancer is often found at an advanced stage in China. Approximately $50 \%$ of patients with advanced gastric cancer die from recurrence and metastasis, even after curative surgery and chemotherapy [2]. Resistance to chemotherapy and limited therapeutic options are the leading causes of mortality among metastatic patients. However, the complex metastatic cascade remains the most poorly understood component of cancer pathogenesis. With regard to the metastatic process, physical translocation of a cancer cell from the primary tumor to the microenvironment of the distant tissue and colonization are the crucial steps [3]. It is widely accepted that activation of epithelial-mesenchymal transition (EMT) enables cancer cells to acquire the ability to invade and disseminate [4]. A subpopulation of tumor cells with self-renewal and multi-lineage differentiation capacity, defined as cancer stem cells(CSCs), has been proven to be essential for successful metastasis formation in a distant organ [5]. An increasing number of studies have provided strong evidences for aberrant activation of EMT and CSC cellular pathways during carcinoma metastasis and progression, so a clear understanding of the molecular mechanisms of EMT program and CSC biology may lead to effective therapies for patients with metastases.

Wnt signaling controls embryogenesis and homeostasis through modulating cellular processes such as proliferation, differentiation, migration, apoptosis and cell polarity. Activating mutations of Wnt pathway have been found in a large number of sporadic tumor types including gastrointestinal cancer, breast cancer and leukemia. Wnt signaling is important for driving self-renewal of cancer stem cells and inducing tumor cell epithelial-mesenchymal transition (EMT) which could promote cancer metastasis and progression [6]. Wnt signaling is commonly divided into canonical and non-canonical signaling based on whether $\beta$-catenin is activated (canonical) and initiated by Wnt ligand binding to the surface Frizzled (FZD) receptors. Frizzled receptors have seven transmembrane domains, intracellular C-terminal with a putative PDZ binding domain and extracellular N-terminal domain, which are responsible for binding to Wnt ligands [7]. Increased levels of FZD receptors have been displayed in many cancers, suggesting that the aberrant activation of Wnt signaling via the receptors is involved in accelerating cancer growth [8]. Frizzled 7, a member of Frizzled family, is able to regulate the transmission of both canonical and non-canonical Wnt signaling pathways. Accumulating evidence shows that FZD7 is involved in the activation of canonical Wnt/ $\beta$-catenin signaling in breast, colorectal and hepatocellular carcinoma (HCC) [9], and it increases RhoA activity in colon cancer, implying the participation in non-canonical Wnt/PCP signaling through RhoA [10]. Recent studies have reported that FZD7 expression is up-regulated in human primary gastric cancer tissues and even in side populations of gastric cancer cell lines $[11,12]$. However, a mechanistic understanding of how FZD7 promotes gastric cancer development and progression is left unanswered by the previous studies.

Here we presented a more extensive analysis of FZD7 in GC and showed that FZD7 was overexpressed in clinical late-stage cases of GC, and thus was correlated with poor patient survival. RNA interference-mediated silencing of FZD7 decreased proliferation, invasion, EMT and the expression levels of CSC markers in gastric cancer cells. Meanwhile, we found that FZD7 mediated CSC self-renewal and EMT in gastric cancer via canonical Wnt signaling pathway.

\section{Methods}

\section{Clinical samples and cell lines}

A total of 251 gastric cancer patients who underwent radical gastrectomy at the First Affiliated Hospital, Sun Yat-Sen University were enrolled in this study. After radical gastrectomy, most of patients received 8 cycles of adjuvant chemotherapy (FLOX: 5 -fluorouracil $500 \mathrm{mg} / \mathrm{m}^{2}$, Calcium Folinate 500 $\mathrm{mg} / \mathrm{m}^{2}$, Oxaliplatin $85 \mathrm{mg} / \mathrm{m}^{2}$ ). The patients included 169 males and 82 females, ranging in age from 19 to 87 years (mean 56 years). All patients were followed up and the average follow-up time was 57.1 months. Histopathologic classification was based on World Health Organization and Japanese Gastric Cancer Association for gastric cancer criteria [13, 14]. Tumor staging was in accordance with the International Union Against Cancer (UICC) recommendation [15]. Clinical and pathological features of these 251 GC patients were summarized in Table S1. Samples used in this study were approved by the Committees for Ethical Review of Research involving human subjects at the First Affiliated Hospital, Sun Yat-Sen University.

Gastric cancer cell lines were kindly provided by Professor Jie Chen from the Department of Gastroenterology, the First Affiliated Hospital of Sun Yat-sen University. The cell lines were maintained in DMEM medium with $10 \%$ fetal bovine serum.

\section{Immunohistochemistry}

IHC staining was performed using the standard streptavidin-biotin-peroxidase complex method. Briefly, $3 \mu \mathrm{m}$ paraffin sections were dewaxed and 
rehydrated using xylol and a descending alcohol series. Endogenous peroxidase activity was blocked with 3\% hydrogen peroxide for 20 minutes. Slides were heated for antigen retrieval in a microwave oven for 10 minutes in $10 \mathrm{mM}$ citrate buffer, $\mathrm{pH}$ 6.0. Sections were incubated with polyclonal rabbit anti-Frizzled 7 antibody (diluted 1:50, Abcam) at $4^{\circ}$ overnight. Nonimmune rabbit sera were included as negative control. EnVision Plus System-HRP (DAKO, Denmark) was used according to manufacturer's instruction, and followed by Mayer's hematoxylin counterstaining.

\section{Evaluation of staining of tissue slides}

Immunostaining of FZD7 was evaluated by a semi-quantitative immunoreactivity scoring system (IRS)[12]. As described in the previous study, both the staining intensity and the percentage of positively stained tumor cells were taken into consideration. Immunostaining index of intensity was estimated as 0 (no immunostaining), 1 (weak), 2 (moderate), and 3(strong). The percentage of immunoreactive cells was scored as 0 (no immunoreactive cells), $1(<10 \%), 2$ (10\% to $50 \%), 3(51 \%$ to $80 \%)$, and 4 (>80\%). Addition of intensity and percentage indexes resulted in an IRS ranging from 0 to 7 for each case. Cases with an IRS of 1 or higher were categorized as positive, and those with an IRS less than 1 were categorized as negative. 10 random fields at $100 \times$ magnification were examined and a minimum of 500 cells or more was counted. The sections were viewed and evaluated by two independent observers without prior knowledge of histopathology data. With discrepant results of the same slide, both observers reviewed again to obtain a consensus.

\section{RNA interference and transfection}

Small interfering RNAs (siRNAs) targeting FZD7 and control siRNA were obtained from Genepharma (Shanghai, China). AGS and HGC27 cells were seeded to be $60 \%$ confluent per well in 6-well plates and transfected with $10 \mu \mathrm{M}$ siRNA using Lipofectamine RNA iMAX reagent (Invitrogen, CA, USA), according to the manufacturer's protocol. After 48 hours, the subsequent experiments were performed. The siRNAs targeting FZD7 were as follows: FZD7-1,5'-CCAACG GCCUGAUGUACUUTT-3';FZD7-2,5'-GCACCAUCC UCUUCAUGGUTT-3';FZD7-3,5'-CCGUCAAGACCA UCACUAUTT-3'.

\section{RNA isolation and quantitative real-time PCR}

RNA was isolated from the cell lines using the RNA isolation reagent Trizol (Invitrogen, NY, USA). Single-stranded cDNA was generated from $1 \mu \mathrm{g}$ total RNA in a $20 \mu \mathrm{L}$ reaction volume with $1 \mu \mathrm{L}$ of the
oligo(dT)12-18 primer $(0.5 \mu \mathrm{g} / \mu \mathrm{L})$ and $1 \mu \mathrm{L}$ of $200 \mathrm{U} / \mu \mathrm{L}$ Revert Aid M-MuLV reverse transcriptase (Thermo Fisher, MA, USA) according to the manufacturer's instructions. The real-time quantitative PCR reaction was performed with the SYBR green detection system (Takara, Japan). GAPDH served as an endogenous control. The relative expression levels were measured by qRT-PCR using CFX96 ${ }^{\mathrm{TM}}$ Real-Time PCR Detection (Bio-Rad, CA, USA). Each of the experiments was performed in triplicate. The primer pairs for each target gene are listed in Table S2.

\section{Western blot analysis}

Total cellular and nuclear proteins were extracted from cultured cells. The protein content was measured using the BCA Protein Assay Kit (KeyGen Biotech, Nanjing, China). Quantified protein lysates were resolved on SDS-PAGE, transferred onto a polyvinylidene fluoride (PVDF) membrane (EMD Millipore, MA, USA), and then blocked with 5\% non-fat milk for 1 hour at room temperature. The membranes were incubated with different optimally diluted primary antibodies at $4^{\circ}$ overnight. After washing with PBS-T, the membrane was incubated for 1 hour with horseradish peroxidase (HRP)-conjugated secondary antibody. The peroxidase activity was detected by the ECL (EMD Millipore, MA, USA) method. This assay was performed in triplicate. GAPDH was used as a housekeeping control. The information about antibodies was provided in Table S3.

\section{Cell proliferation and growth}

Cell proliferation was measured by XTT assay (Dojindo, Japan). According to the manufacturer's protocol, cells in logarithmic growth phase were plated in 96-well plates at the appropriate density. Six duplicate wells were set for each group with a negative control. $10 \mu \mathrm{L}$ of the XTT substrate was then added into each well, the plates were incubated at $37^{\circ}$ for 3 hours, and the OD values were measured with microplate reader at a wavelength of $490 \mathrm{~nm}$. XTT assays were performed once a day until day 5 . The growth curve was constructed by plotting absorbance against time. For foci formation assay, $2 \times 10^{2}$ cells were plated in $3.5 \mathrm{~cm}$ culture plate in triplicates with DMEM medium containing 10\% FBS. Surviving colonies (>50 cells per colony) were stained with $0.05 \%$ crystal violet and counted after one week in culture.

\section{Cell migration and invasion}

For wound healing assay, cells were seeded in 6 well plates and cultured until confluent. Scratch wounds were made using a sterile $10-\mu \mathrm{L}$ pipette tip to 
create a straight cell-free line. Migration photos were captured at 0 and 48 hour after scratching. Transwell invasion assay was performed using polyethylene terephthalate-based migration chambers and $\mathrm{BD}$ BioCoat Matrigel Invasion Chambers (Becton Dickinson Labware, USA) according to the manufacturer's instructions. Cells in serum-free media were distributed into the inserts. Equal amounts of growth media were placed into the wells. After overnight culture, the chamber membrane was stained with $50 \%$ methanol blue/ethanol overnight.

\section{Flow cytometry}

To identify gastric CSCs, APC-labeled anti-human CD24 antibody (Miltenyi, BergischGladbach, Germany), PE-labeled anti-human CD133 (Miltenyi, Bergisch-Gladbach, Germany) and APC/ Cy7- labeled anti-human CD44 (eBiosciences, CA, USA) were used. Background signals were established using control cells incubated with isotype-specific IgGs. Fluorescence intensity was analyzed using the Gallios Flow cytometer (FlowCount, Beckman Coulter, CA, USA) and the data were analyzed using KALUZA software (Ver. 2.0.1).

\section{Spheroid formation assay}

AGS and HGC27 cells were seeded into 6-well poly HEMA-coated plates (Sigma-Aldrich, St Louis, MO) and cultured for 14 days in DMEM/F12 medium supplemented with 20ml/L B27 (Invitrogen Carlsbad, CA, USA), 20ng/mL EGF (Invitrogen Carlsbad, CA, USA) and $10 \mathrm{ng} / \mathrm{mL}$ b-FGF (Invitrogen Carlsbad, CA, USA). Cells were replenished with fresh medium every second day. The spheres in the suspension culture were observed under microscope.

\section{Immunofluorescence staining}

Slides were fixed in $4 \%$ formaldehyde in PBS for 15 minutes, rinsed three times in PBS, and then permeabilized with $0.1 \%$ TritonX-100 in PBS for 20 minutes. Slides were then blocked with 3\% BSA in PBS for 1 hour at room temperature followed by incubation with the primary antibody at $4^{\circ}$ overnight and the secondary antibody for 1 hour at room temperature. Slides were mounted with Vectashield mounting medium and images were taken with Olympus BX53 microscope. Goat anti-rabbit secondary antibodies conjugated to Alexa Fluor 594 (A-11072) (Invitrogen Carlsbad, CA, USA) were purchased from Invitrogen.

\section{Statistical analysis}

All statistical analyses were conducted using commercially available software (SPSS version 18.0; SPSS, Inc, Chicago, IL). The significance of associations between FZD7 immunostaining patterns and clinicopathologic characteristics was tested using the Chi-square test or the Fisher exact test. Student's $t$-test was used to evaluate the data from cell growth, foci formation, wound healing, transwell, western blot and qPCR assays. Survival analysis was performed using Kaplan-Meier method for univariate analysis with a log-rank test for significance and Cox regression for multivariate analysis. The value of $P<0.05$ was considered statistically significant.

\section{Result}

\section{The expression of FZD7 is up-regulated in gastric cancer and associated with advanced tumor stages and poor survival}

To investigate the FZD7 expression level in gastric cancer, we first analyzed FZD7 mRNA expression in human gastric cancer and normal gastric tissues by querying the ONCOMINE database. GSE27342 expression dataset from 80 gastric cancer and 80 normal gastric tissue specimens, GSE13861 expression dataset from 31 gastric cancer and 19 normal gastric tissue specimens and GSE19826 expression dataset including 12 gastric cancer, 3 gastric mucosa and 12 whole gastric tissue specimens were chosen in our study. These datasets indicated that FZD7 mRNA expression was significantly up-regulated in gastric cancers in comparison with normal gastric tissues (Figure 1A). To identify the protein expression of FZD7 in GC samples, FZD7 was detected by IHC in 251 primary GC specimens and 60 non-neoplastic tissues. In 60 non-neoplastic tissues, FZD7 was expressed in cytoplasm and occasionally at cell membrane in epithelial cells. The negative staining was found in 20 cases, weak or moderate staining (IRS $\leq 3$ ) in 37 cases and strong staining (IRS $>4$ ) in only 3 cases. Since the levels of FZD7 expression in most normal gastric tissues were negative or weak (Figure 1B), IRS $\leq 3$ was defined as normal expression level and IRS $>4$ was considered as over-expression in the present study. Using this criteria, over-expression of FZD7 was observed in $100 / 251(47.8 \%)$ of the gastric cancers, which was significantly higher than that in non-neoplastic tissues $(P<0.0001$, Figure 1C and 1D). The association of FZD7 over-expression with the clinicopathologic characteristics of the patient cohort was furtherly evaluated, which was summarized in Table 1 . Over-expression of FZD7 was not associated with patient's age, sex and histologic type. Intriguingly, over-expression of FZD7 was significantly correlated with tumor invasion $(P<0.0001)$, lymphatic metastasis $(P<0.0001)$, distant organ metastasis $(P<0.0001)$ and late TNM stages $(P<0.0001)$. 
A
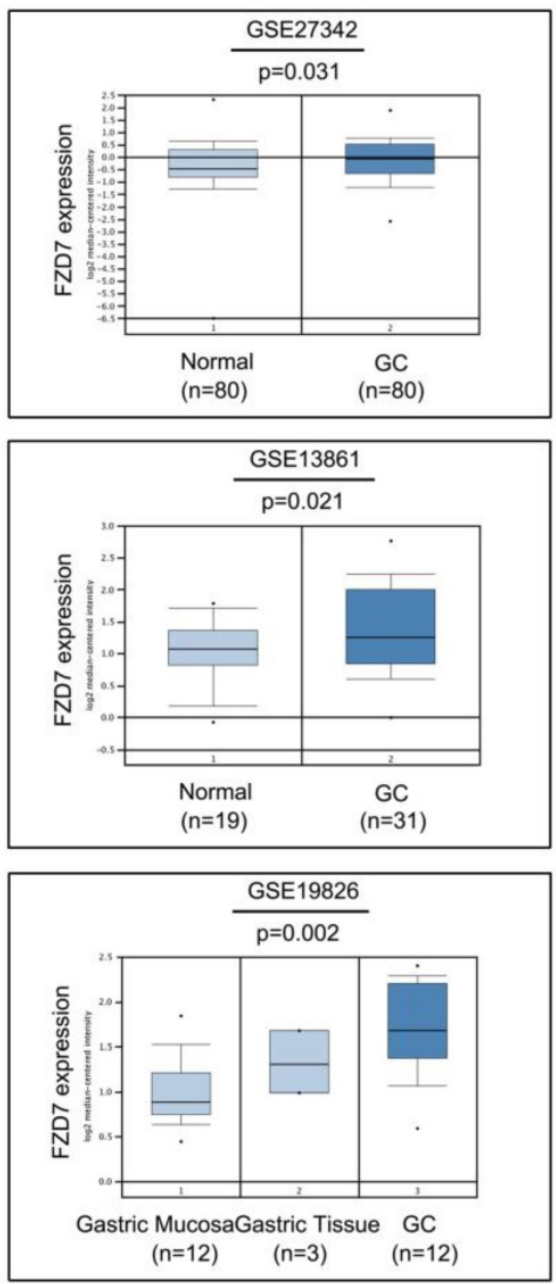

E

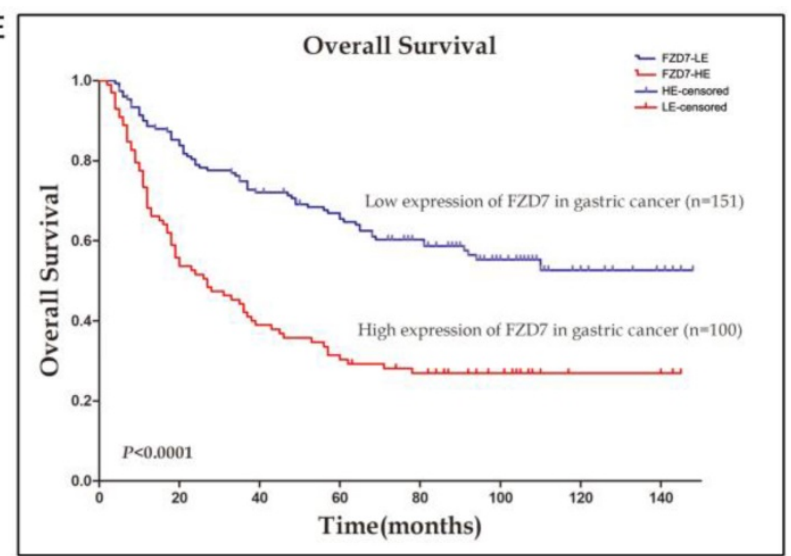

B

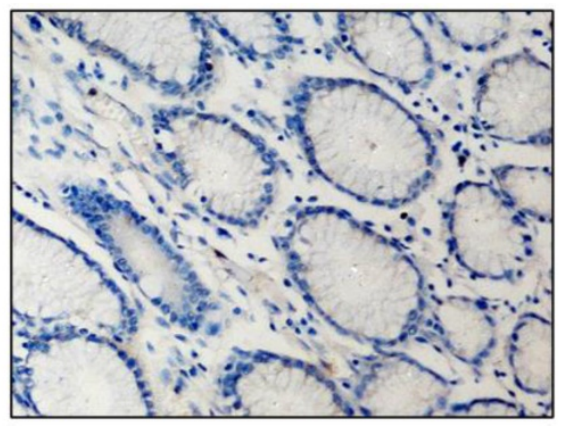

C

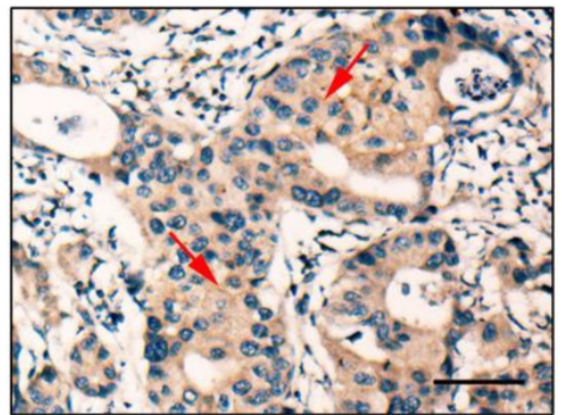

D

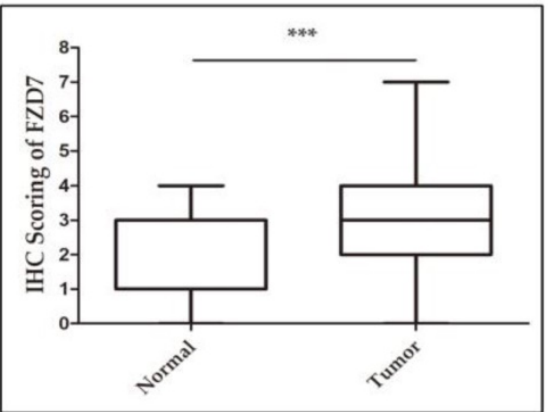

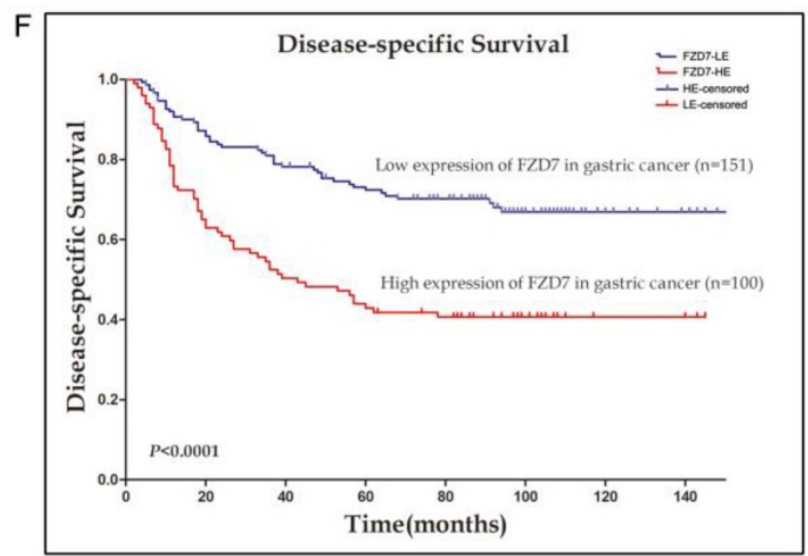

Fig.1 The expression of FZD7 is up-regulated in gastric cancer and associated with invasion, metastasis, advanced tumor stages and poor survival (A) Analyses of GSE27342, GSE13861 and GSE19826 datasets in ONCOMINE database revealed significant increases of FZD7 mRNA expression in gastric cancer tissues versus normal tissues. (B) Representative of negative expression of FZD7 in normal gastric mucosa (Original magnification, $\times 200$, scale bars: 100 $\mu m$ ). (C) Representative of over-expression of FZD7 in a GC specimen with staining index 6. Red arrows indicated positive staining for FZD7 in cytoplasm and membrane.

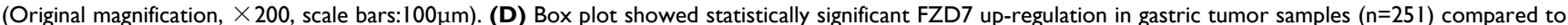
normal gastric tissues $(n=60)(* * *$ indicates $P<0.001)$. (E)(F) Kaplan-Meier analysis for the association of FZD7 expression with overall $(E)$ and gastric cancer-specific survival (F) in 251 gastric cancers.

The potential correlation between up-regulation of FZD7 and GC prognosis was also addressed in this study. The overall 5-year survival rate in these 251 GC patients was $51.5 \%$, with a median survival time of 53 months. In GC patients with high FZD7 expression, the overall 5-year survival rate $(30.3 \%$, with a median 
of 23.5 months) was significantly lower than that in GC patients with down-expression of FZD7 (65.4\%, with a median of 77 months, $P<0.001)$. The Kaplan-Meier estimates of survival stratified by FZD7 expression were shown in Figure 1E and 1F. Visual inspection of the Kaplan-Meier curves suggested that both overall and cancer-special survival times of patients with low expression of FZD7 were significantly longer than that of patients with FZD7 over-expression $(P<0.0001)$. Univariate Cox regression analysis showed that up-regulation of FZD7 and TNM stage were significantly associated with patient poor survival. Multivariate Cox regression indicated that TNM stage and up-regulation of FZD7 were independent prognostic factors in GC patients (Table 2).

Table 1. The association between FZD7 expression and clinicopathological features in 251 gastric cancers.

\begin{tabular}{|c|c|c|c|}
\hline $\begin{array}{l}\text { Clinicopathological } \\
\text { features }\end{array}$ & Total case & $\begin{array}{l}\text { Over-expression of } \\
\text { FZD7 }\end{array}$ & $P$ \\
\hline No. of patients & 251 & $100 / 251(39.8 \%)$ & \\
\hline Age (mean 56 years) & & & 0.571 \\
\hline$\leq 56$ & 126 & $48 / 134(38.1 \%)$ & \\
\hline$>56$ & 125 & $52 / 129(41.6 \%)$ & \\
\hline Sex & & & 0.119 \\
\hline Female & 82 & $27 / 82(32.9 \%)$ & \\
\hline Male & 169 & $73 / 169(43.2 \%)$ & \\
\hline Histological type1 & & & 0.295 \\
\hline WA & 78 & $29 / 78(37.2 \%)$ & \\
\hline PA & 131 & $58 / 131(44.3 \%)$ & \\
\hline MA & 13 & $2 / 13(15.4 \%)$ & \\
\hline SRC & 22 & $9 / 22(40.9 \%)$ & \\
\hline $\mathrm{UC}$ & 7 & $2 / 7(28.6 \%)$ & \\
\hline Tumor stage & & & $<0.0001$ \\
\hline pT1- pT2 & 48 & $7 / 48(14.6 \%)$ & \\
\hline pT3- pT4 & 203 & $93 / 203(45.8 \%)$ & \\
\hline Lymph-node metastasis & & & $<0.0001$ \\
\hline pNo & 123 & $25 / 123(20.3 \%)$ & \\
\hline $\mathrm{pN}+$ & 128 & $75 / 128(58.6 \%)$ & \\
\hline Organ metastasis & & & $<0.0001$ \\
\hline M0 & 176 & $53 / 176(30.1 \%)$ & \\
\hline M1 & 75 & $47 / 75(62.7 \%)$ & \\
\hline TNM Stage & & & $<0.0001$ \\
\hline I & 46 & $6 / 46(13.0 \%)$ & \\
\hline II & 75 & $20 / 75(26.7 \%)$ & \\
\hline III & 54 & $25 / 54(46.3 \%)$ & \\
\hline IV & 76 & $49 / 76(64.5 \%)$ & \\
\hline
\end{tabular}

${ }^{1}$ Histological type: $\mathrm{WA}$, well/moderately differentiated adenocarcinoma; $\mathrm{PA}$ poorly differentiated adenocarcinoma; MA, mucinous adenocarcinoma; SRC, signet ring cell carcinoma; UC, undifferentiated

\section{Knockdown of FZD7 suppressed gastric cancer cell growth, migration and invasion}

To choose cell lines for studying the functional role of FZD7 in GC tumorigenesis, we examined the protein levels of FZD7 by western blotting in seven GC cell lines (NCI-N87, MKN28, SGC7901, AGS, MGC803, HGC27, BGC823) and one normal gastric epithelial cell line (GES-1). In these cell lines, the highly invasive human GC cell lines AGS, MGC803 and HGC27, expressed high levels of FZD7 (Figure 2A). Three different FZD7 siRNAs (FZD7-1, FZD7-2, and FZD7-3) were used to knockdown FZD7 in AGS and HGC27 cells and a non-targeted siRNA was used as negative control. We achieved approximately $80 \%$ knockdown in AGS and HGC27 cells detected by qPCR and western blotting (Figure S1). XTT assay showed that the cell growth rates in FZD7-repressing cells were significantly decreased more than 2 folds compared with the control cells after 5 days in culture $(P<0.001$, Figure 2B). In foci formation assay, the number and the size of the colonies formed from FZD7-scilencing cells were significantly reduced $(P<0.001$, Figure $2 \mathrm{C})$. Wound healing assay indicated that ablation of FZD7 delayed primary wound closure $(P<0.001$, Figure 2D). Transwell assay showed that the migratory and invasive abilities were significantly decreased in FZD7-silencing cells compared with their corresponding control cells $(P<0.01$, Figure 2E). The results illustrated that FZD7 could promote tumor growth and invasion.

\section{Ablation of FZD7 decreased epithelial-mesenchymal transition (EMT) and MMP-7 expression}

EMT is one of the key processes in tumor invasion and metastasis. Accumulating evidence demonstrates that activation of Wnt signaling can drive a transcriptional program and promote EMT in cancers [16]. To determine the effect of FZD7 on EMT, the expression levels of EMT markers and EMT-related transcription factors were examined in FZD7-silencing and control cells. Western blot analysis showed that epithelial marker E-cadherin was increased, while mesenchymal markers $\mathrm{N}$-cadherin, fibronectin and vimentin were decreased in FZD7-silencing cells compared with control cells (Figure 3A). In line with the western blot data, qPCR analysis indicated that the mRNA expression level of epithelial marker was up-regulated, but mesenchymal markers were down-regulated in FZD7-silencing cells (Figure 3B). Snail, one of EMT-related transcription factors, was simultaneously attenuated when FZD7 was down-regulated. Matrix metalloproteases (MMPs) are proteolytic enzymes required for the pericellular degradation of basement membrane and interstitial extracellular matrix (ECM), and contribute to cancer invasion and growth. MMP-7 is one of the key MMPs responsible for EMT in gastric adenocarcinoma [17]. As expected, the expression level of MMP-7 was also decreased in FZD7-silencing cells compared with control cells (Figure 3A and 3B). Collectively, these data strongly suggested that FZD7 might be a positive mediator of EMT in gastric cancer cells. 
A

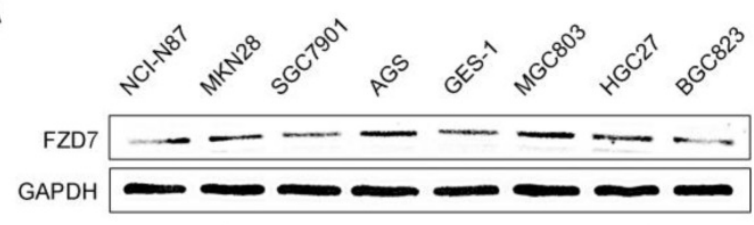

B

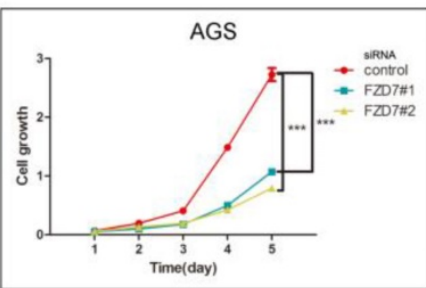

C

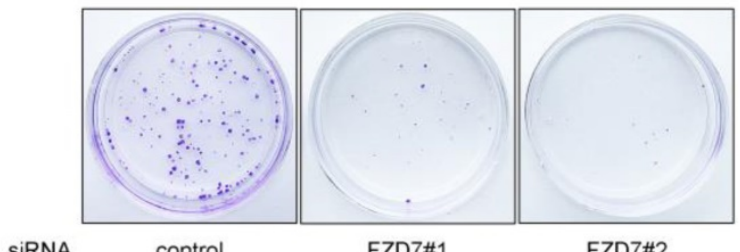

siRNA

AGS

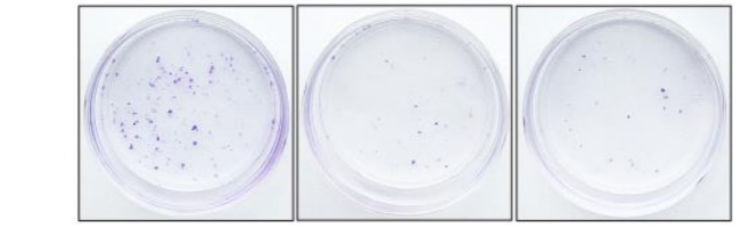

SiRNA

control

FZD7\#1

HGC27

D

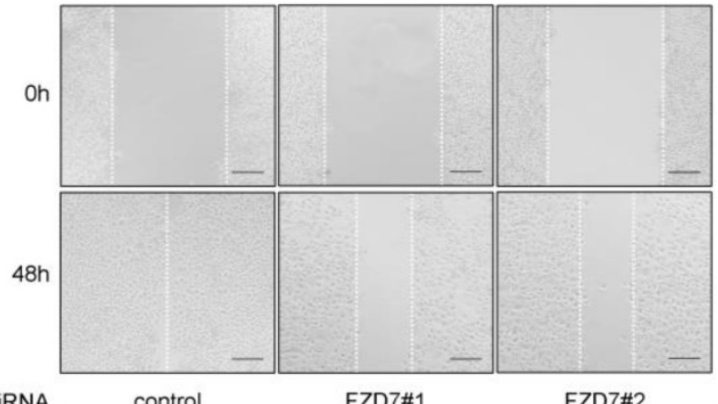

siRNA

control

FZD7\#1

E

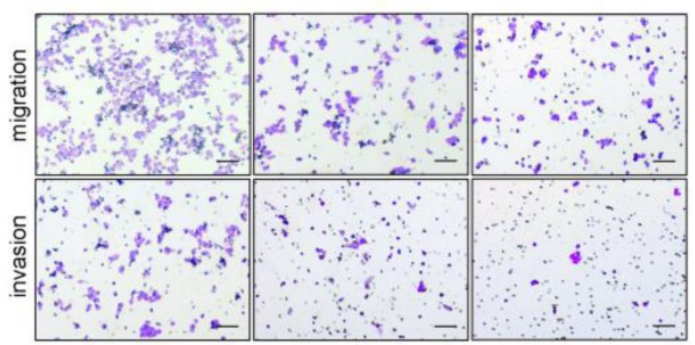

siRNA

FZD7\#1
FZD7\#2

FZD7\#2

(1)

AGS
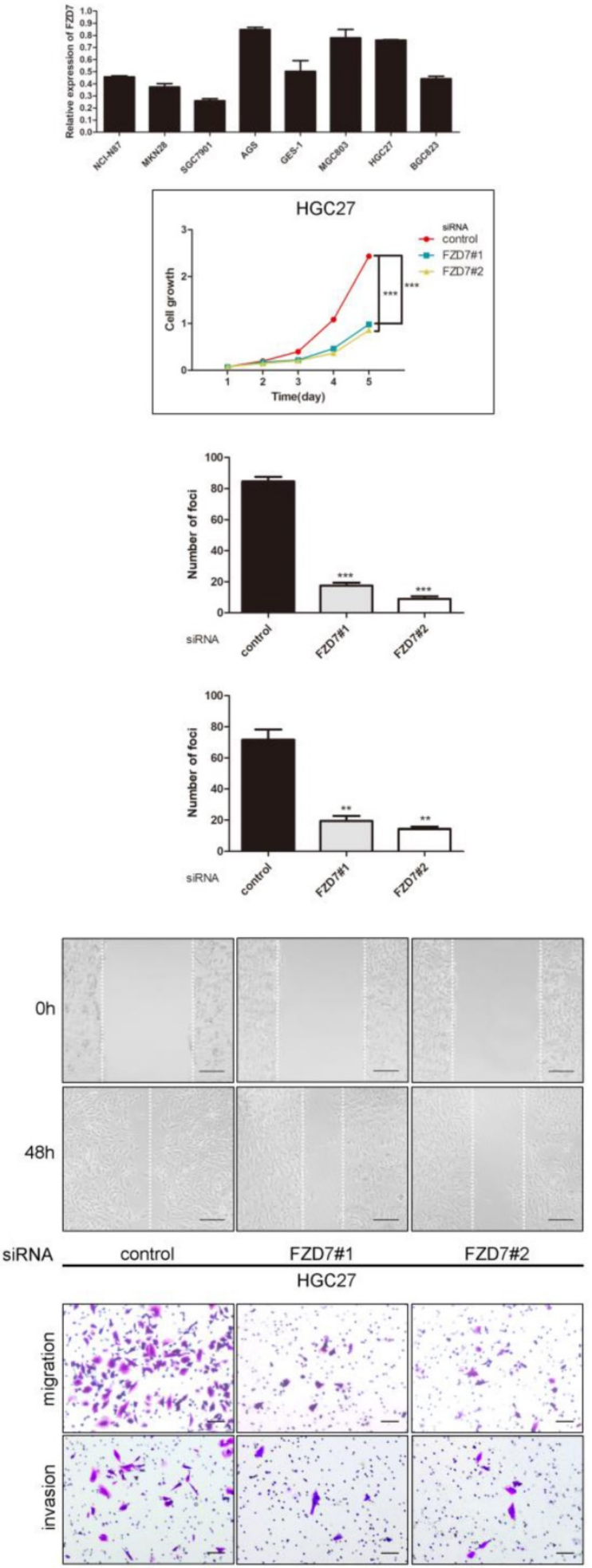

siRNA control FZD7\#1 FZD7\#2

Fig.2 Knockdown of FZD7 suppressed gastric cancer cell growth, migration and invasion (A) Western blot analysis of expression of FZD7 in gastric cancer cell lines. Quantifications of endogenous FZD7 expression were shown in the right panel. Intensities of bands were analyzed by the Macintosh densitometry program Image J (NIH, Bethesda, MD). Values was reflected as the mean \pm SD of three independent experiments. (B) XTT assay showed that ablation of endogenous FZD7 inhibited cell proliferation in AGS and HGC27. The results were expressed as the mean \pm SD of three independent experiments. (*** indicates $P<0.001$ in independent Student's t-test.) (C) Foci formation assay indicated that silencing of FZD7 inhibited cell colony formation. Quantitative analyses of foci numbers were shown in the right panel. Values was reflected as the mean \pm SD of three independent experiments. ( $* * *$ indicates $P<0.001$ in independent Student's t-test.) (D) Wound healing assay showed that FZD7 depletion inhibited cell migration (Scale bars: $100 \mu \mathrm{m})$. Representative images were taken at $0 \mathrm{~h}$ and $48 \mathrm{~h}$ after scratching. (E) Transwell assay indicated that FZD7 ablation repressed cell invasion (Scale bars:100 $\mu \mathrm{m}$ ). Values were reflected as the mean \pm SD of three independent experiments. 
A

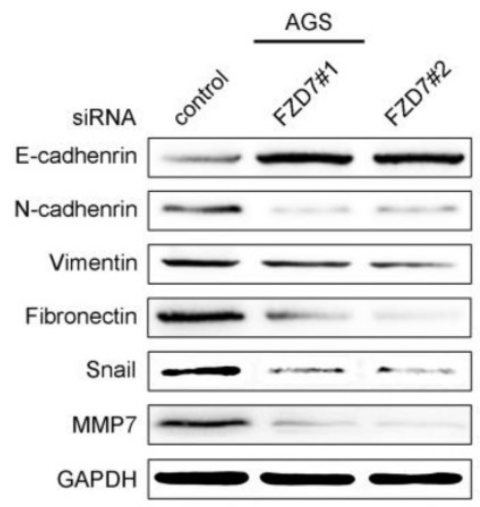

B

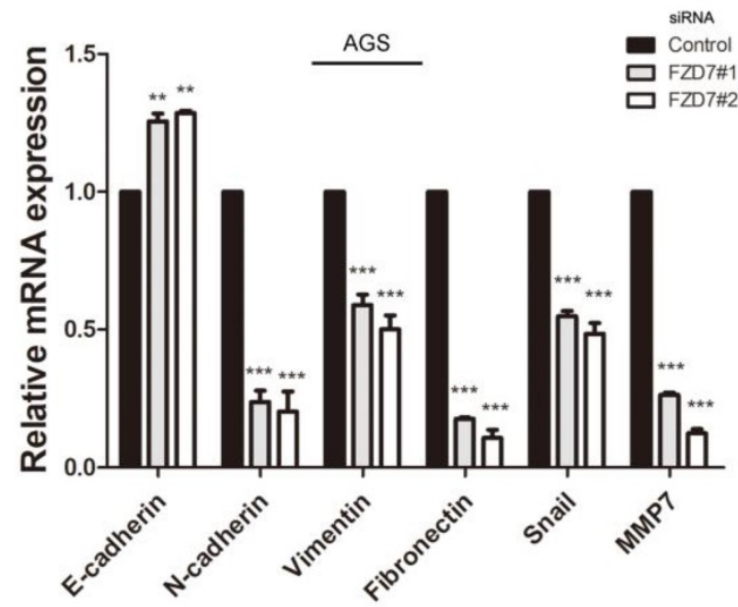

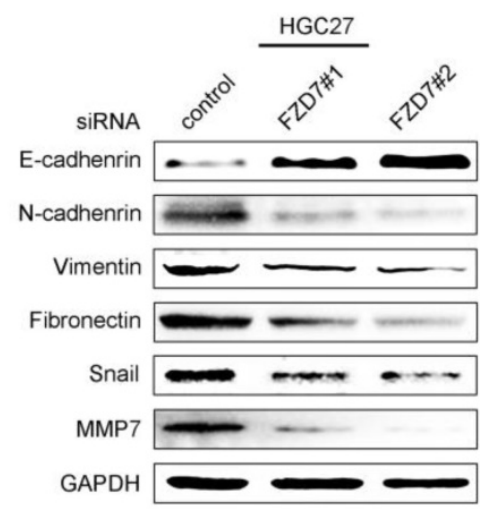

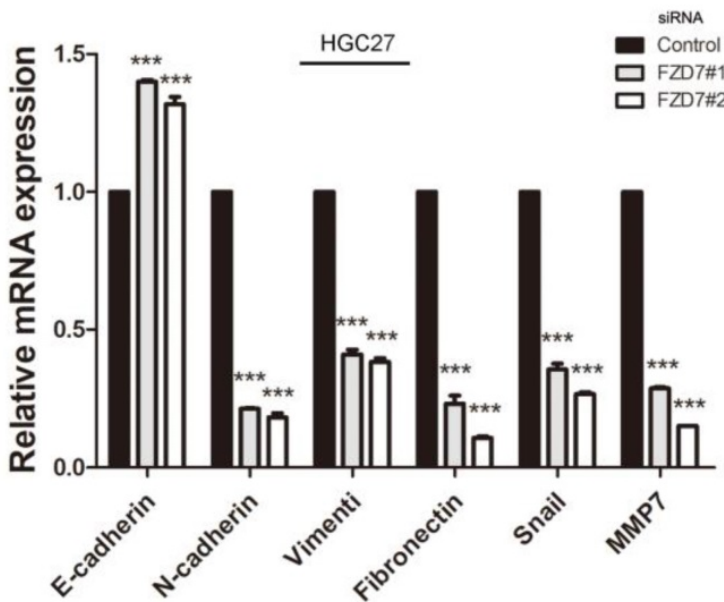

Fig.3 Ablation of FZD7 decreased epithelial-mesenchymal transition (EMT) and MMP7 expression (A) Western blot analysis showed that ablation of endogenous FZD7 caused an increase of E-cadherin expression and decreases of mesenchymal cell marker expression (vimentin, fibronectin and $\mathrm{N}$-cadherin), snail and MMP7 in FZD7-silencing cells compared with their respective control cells. GAPDH was used as a loading control. (B) Quantitative real-time PCR analysis showed that silencing of FZD7 up-regulated mRNA expression of E-cadherin and inhibited mRNA expression of N-cadherin, vimentin, fibronectin, snail and MMP7. (***indicates $P<0.001$, independent Student's t-test).

\section{Down-regulation of FZD7 suppressed stem cell-like properties of gastric cancer cells}

Wnt pathway controls the activity of stem cells during embryonic development, adult homeostasis, and tissue regeneration. Particularly, FZD7-dependent enhancement of Wnt signaling facilitated tumor initiating activity of the basal subtype of breast cancer [18]. We supposed that FZD7 might have an effect on stemness of gastric cancer cells. Firstly, we used serum free suspension spheroid culture to enrich the potential cancer stem cell (CSC) subpopulations. Spheres were generated from a minority of floating cells after 14-day suspension culture (Figure 4A). We subsequently assessed the expression levels of stemness-associated genes through qPCR in spheroid cells from gastric cancer cell lines AGS and HGC27 and adherent parental cells. The results showed that stemness genes (Nanog and Oct-4), multiple drug-resistant transporter gene (ABCG2) and surface antigens associated with cancer stem cells (CD24,
CD44 and CD133) were up-regulated in spheroid cells (Figure 4B). Flow cytometry analysis showed that the populations of CD44+/CD24+ and CD44+/CD133+ cells were enriched and the mean fluorescence intensities of the CSC surface markers were higher in spheroid cells (Figure 4C and 4D). CD44+/CD24+ expression was found in $48.71 \%$ of AGS spheroid cells, and $12.84 \%$ of adherent parental cells $(P<0.05)$. The CD44+/CD133+ cells accounted for $63.37 \%$ in AGS spheroid cells and only $0.02 \%$ in adherent cells $(P<0.001)$. The percentage of CD44+/CD24+ cells was $85.93 \%$ in spheres from HGC27 cell line and $16.91 \%$ in adherent cells $(P<0.001)$. The population of CD44+/CD133+ cells was around $85.16 \%$ and $0.89 \%$ in HGC27 spheroid cells and adherent cells respecttively $(P<0.001)$. Next, we investigated expression of FZD7 in spheroid and adherent cells through qPCR and western blot. The results showed that expression of FZD7 was significantly increased in spheres compared with adherent cells (Figure 4E and 4F). 
A
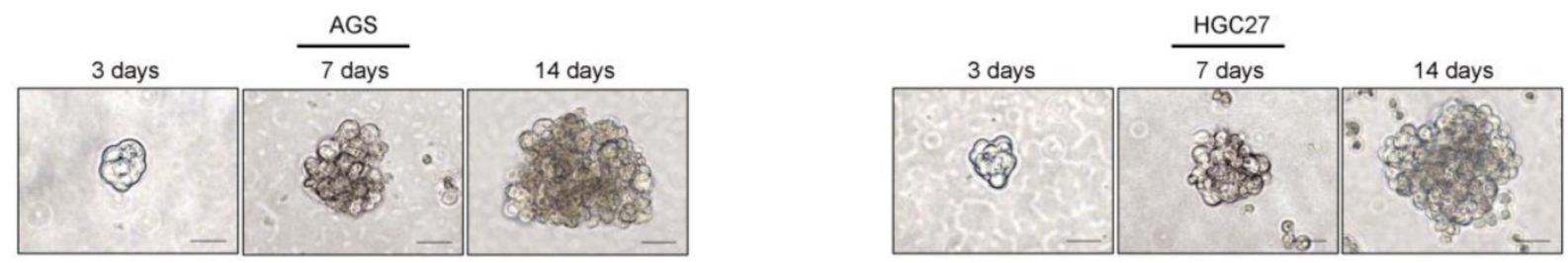

B
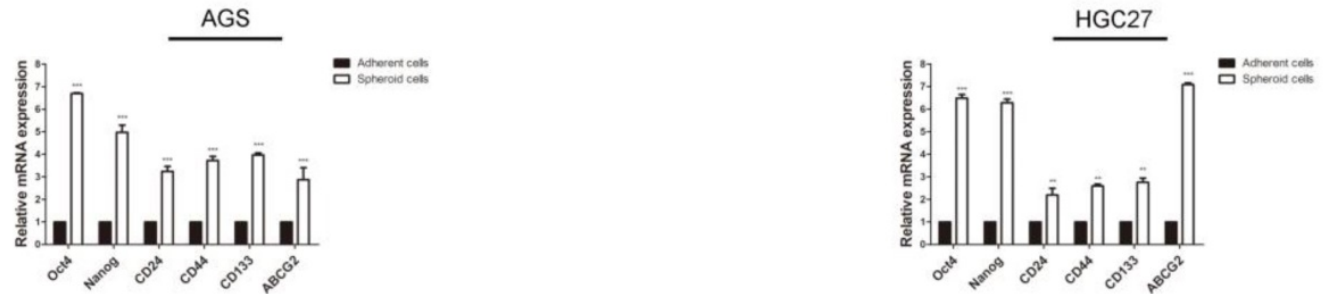

C
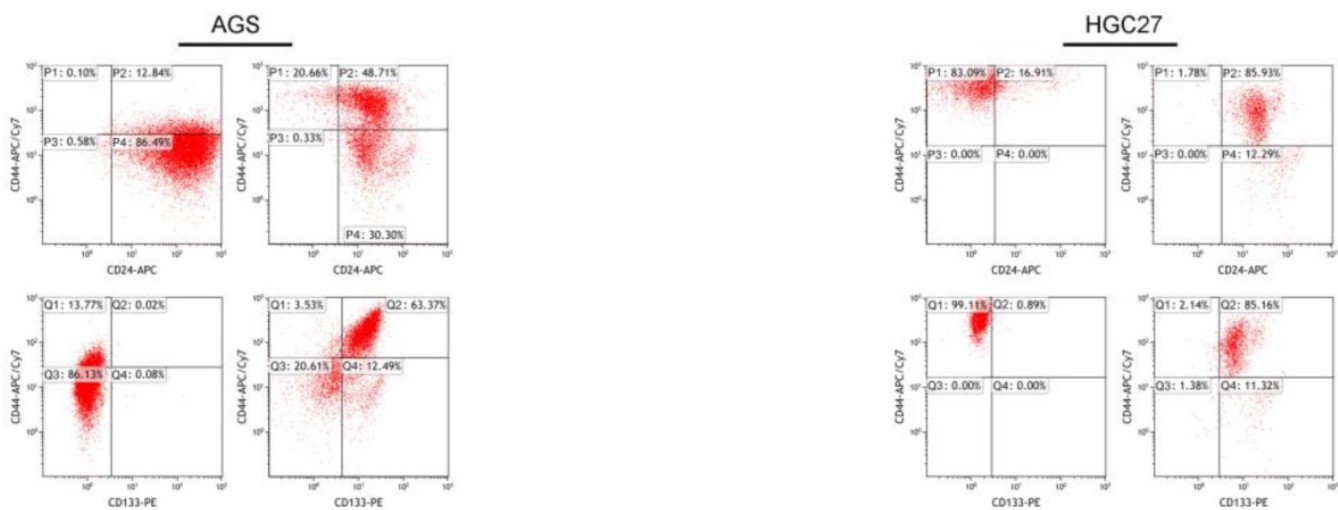

D

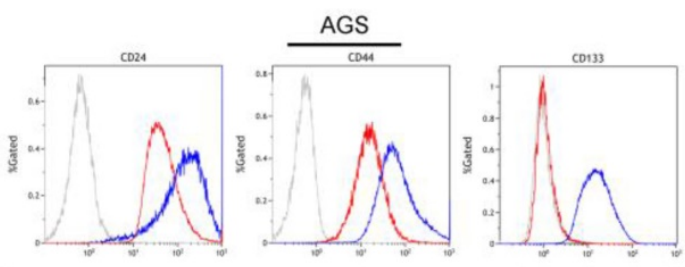

\begin{tabular}{|l|c|c|c|}
\hline AGS & CD24 MFI & CD 44 MFI & CD133 MFI \\
\hline - Negative control cells & $0.65 \pm 0.29$ & $0.52 \pm 0.06$ & $0.92 \pm 0.06$ \\
\hline - Adherent cells & $42.4 \pm 6.42$ & $15.59 \pm 4.89$ & $1.04 \pm 0.23$ \\
\hline - Spheroid cells & $151.1 \pm 7.76 *$ & $58.94 \pm 6.35 *$ & $15.48 \pm 0.39-\cdots$ \\
\hline
\end{tabular}

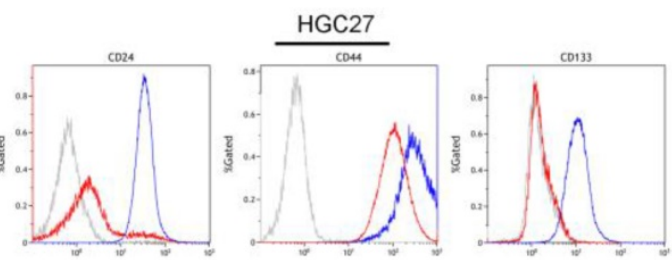

\begin{tabular}{|l|l|c|c|}
\hline HGC27 & CD24 MFI & CD44 MFI & CD133 MFI \\
\hline — Negative control cells & $0.65+0.01$ & $0.63 \pm 0.1$ & $1.32+0.14$ \\
\hline - Adherent cells & $1.42 \pm 0.19$ & $105.17 \pm 1.91$ & $1.49 \pm 0.14$ \\
\hline - Spheroid cells & $34.5+0.38--$ & $311.57 \pm 2.66-$ & $10.83 \pm 1.88--$ \\
\hline
\end{tabular}

E

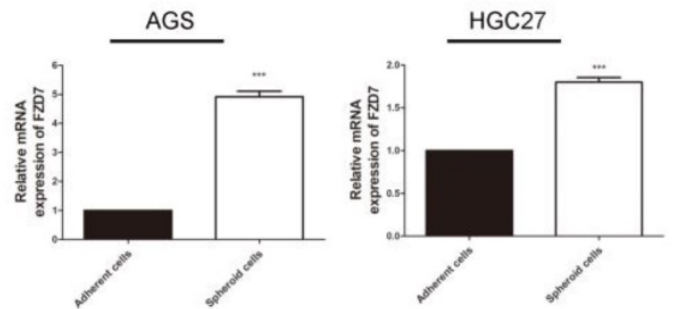

F

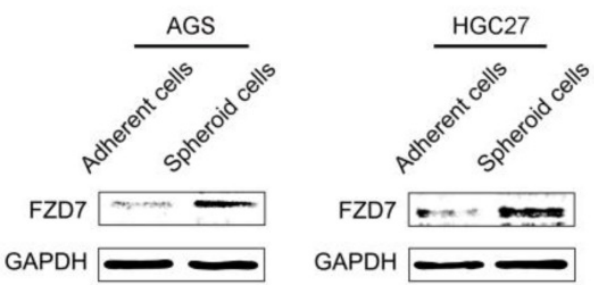

Fig.4 The endogenous expression of FZD7 was significantly increased in cancer stem cell-enriched spheres (A) Spheroid cells were derived from gastric cancer cell lines AGS and HGC27 by serum free culture(SFM) on ultra-low attachment plates. Images were taken at the indicated time points (Scale bars:

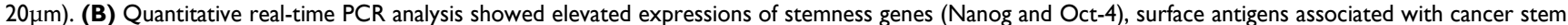
cells (CD24, CD44 and CD133) and multiple drug-resistant transporter gene (ABCG2) in spheroid cells. ( $* *$ indicates $P<0.01$; $* * *$ indicates $P<0.001$, independent Student's t-test). (C)(D) Flow cytometry analysis showed that the populations of CD44+/CD24+ and CD44+/CD133+ cells were enriched and the mean fluorescence intensities of the markers were higher in spheroid cells $(P<0.001)$. $X$ axis, $C D 24$ intensity or $C D 133$ intensity; $Y$ axis, $C D 44$ intensity; $P 1$ : $C D 44$ positive and CD24 negative; P2: CD44 positive and CD24 positive; P3: CD44 negative and CD24 negative; P4: CD44 negative and CD24 positive; Q1: CD44 positive and CD133 negative; Q2: CD44 positive and CD133 positive; Q3: CD44 negative and CDI33 negative; Q4: CD44 negative and CD133 positive. (E)(F) Western blot analysis and quantitative real-time PCR analysis showed that the endogenous expression FZD7 was significantly increased in spheroid cells $(* * i n d i c a t e s ~ P<0.01$; ***indicates $P<0.001$, independent Student's t-test). 
A

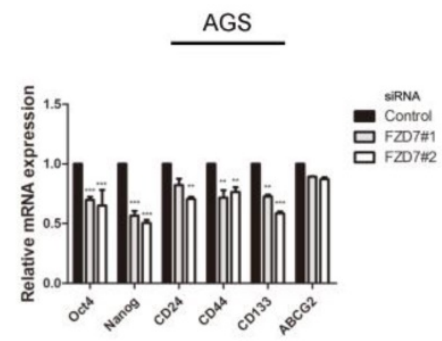

B

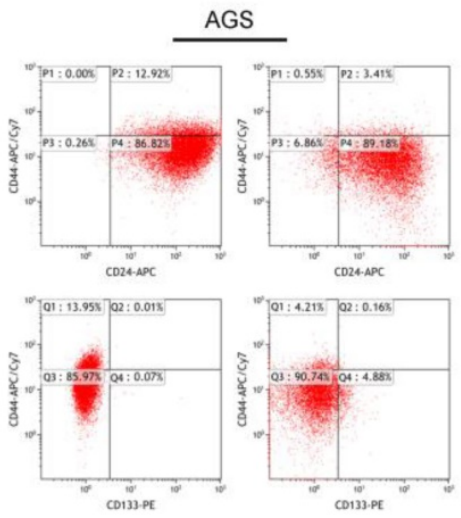

C

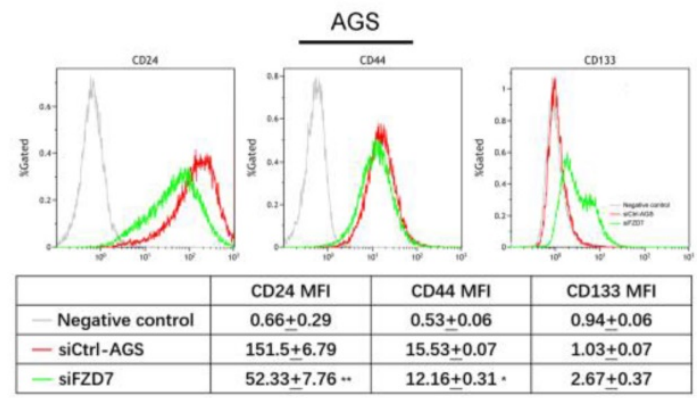

HGC27
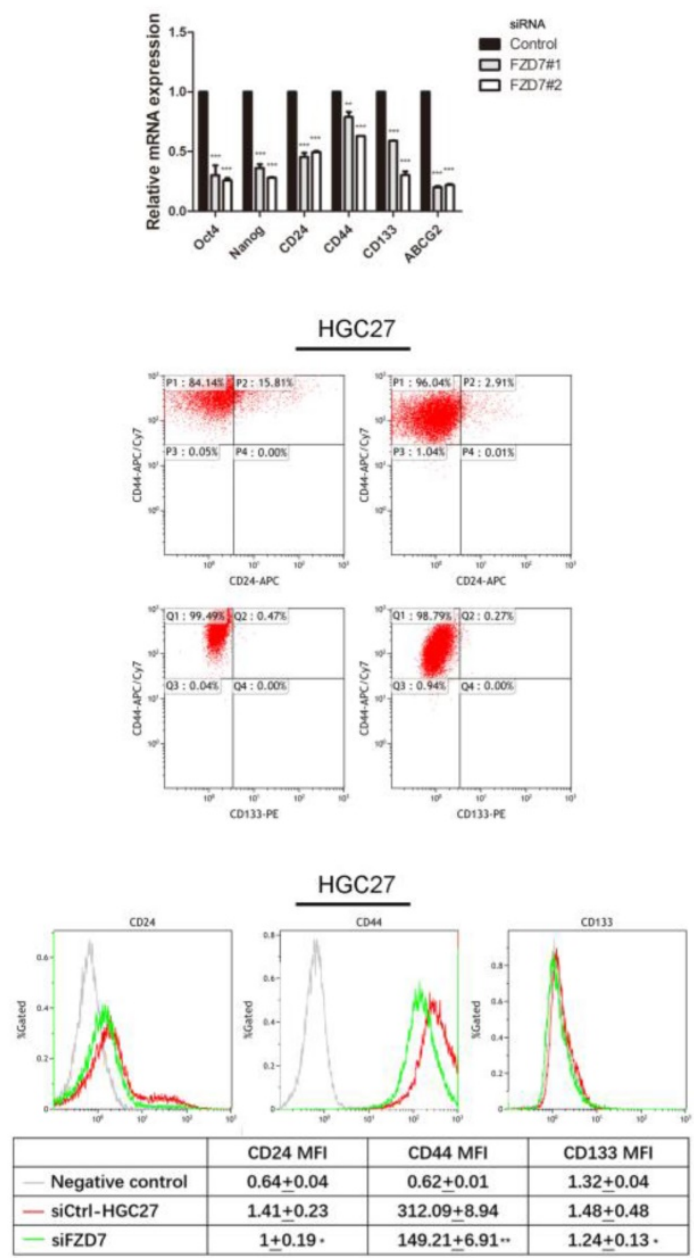

Fig.5 Downregulation of FZD7 expression attenuated stemness of gastric cancer cells. (A) Quantitative real-time PCR analysis showed that the expressions of Nanog, Oct-4, CD24, CD44, CD133 and ABCG2 were down-regulated in FZD7-silencing cells compared with their control cells (*indicates $P<0.05$; **indicates $P<0.01$; ***indicates $P<0.001$, independent Student's t-test). (B)(C) Flow cytometry analysis showed that FZD7-silencing cells exhibited lower levels of expression of CD24, CD44 and CD133 and lower mean fluorescence intensities compared with their control cells $(P<0.05)$. $X$ axis, $C D 24$ intensity or CD133 intensity; $Y$ axis, CD44 intensity; P1: CD44 positive and CD24 negative; P2: CD44 positive and CD24 positive; P3: CD44 negative and CD24 negative; P4: CD44 negative and CD24 positive; Q1: CD44 positive and CD133 negative; Q2: CD44 positive and CD133 positive; Q3: CD44 negative and CD133 negative; Q4: CD44 negative and CD133 positive.

Furthermore, we knocked down FZD7 in AGS and HGC27 cells by siRNAs and evaluated the expression levels of stemness-associated genes through qPCR. The results showed that depletion of FZD7 down-regulated expression of stemness genes (Nanog and Oct-4), surface antigens associated with cancer stem cells (CD24, CD44 and CD133) and multiple drug-resistant transporter gene (ABCG2) (Figure 5A). Flow cytometry analysis showed that FZD7-silencing cells exhibited lower levels of CD24, CD44 and CD133 expressions compared with control cells. In AGS cells, the percentage of cells with CD44+/CD24+ markers accounted for $3.41 \%$ in FZD7-silencing cells and $12.92 \%$ in control cells $(P<0.05)$. In HGC27 cells, the percentages of CD44+/ CD24+ cells were decreased from $15.81 \%$ in control cells to $2.91 \%$ in FZD7-silencing cells $(P<0.05)$.
Although the population of CD44+/CD133+ positive cells was reduced slightly, lower mean fluorescence intensities of CD44, CD24 and CD133 were found in FZD7 deprived cells ( $P<0.05$, Figure 5B and $5 C)$. These results suggested that FZD7 may regulate gastric cancer stem cell function.

\section{Knockdown of FZD7 inhibited Wnt/ $\beta$-catenin signaling pathway in gastric cancer cells}

FZD7 is known as the ligand receptor in Wnt/ $\beta$-catenin signaling pathway. We presumed that FZD7 may promote EMT and CSC activity through canonical Wnt $/ \beta$-catenin pathway. To explore the hypothesis, the cellular levels of $\beta$-catenin and canonical Wnt pathway target genes c-Myc and Cyclin D1 were detected. We observed that the accumulations of $\beta$-catenin in both nucleus and 
cytoplasm were strongly attenuated in FZD7-silencing cells compared with control cells by immunofluorescence staining (Figure 6A and 6B). By western blot analysis, $\beta$-catenin in nucleus was significantly reduced when FZD7 was knocked down. Consistent with the cellular decrease of $\beta$-catenin in
FZD7-silencing cells, the expressions of canonical Wnt pathway target genes c-Myc and Cyclin D1 were diminished by FZD7 inhibition (Figure 6C). These results revealed that FZD7 could induce gastric cancer cell invasion and metastasis via canonical Wnt signaling.
A

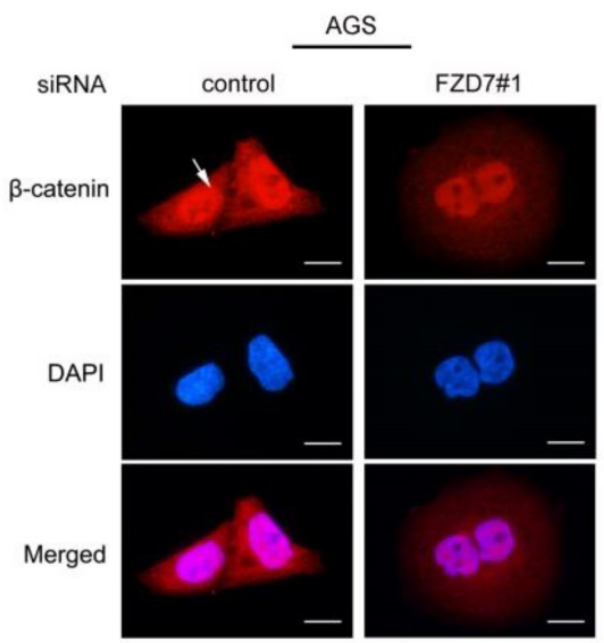

B

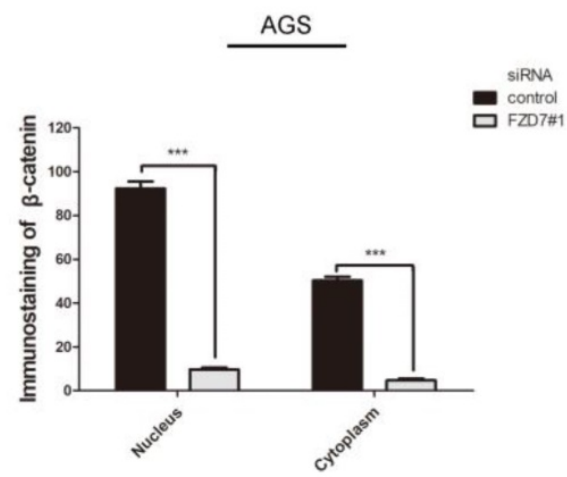

C

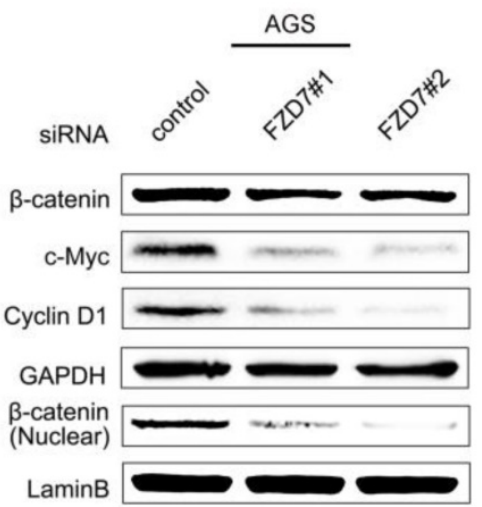

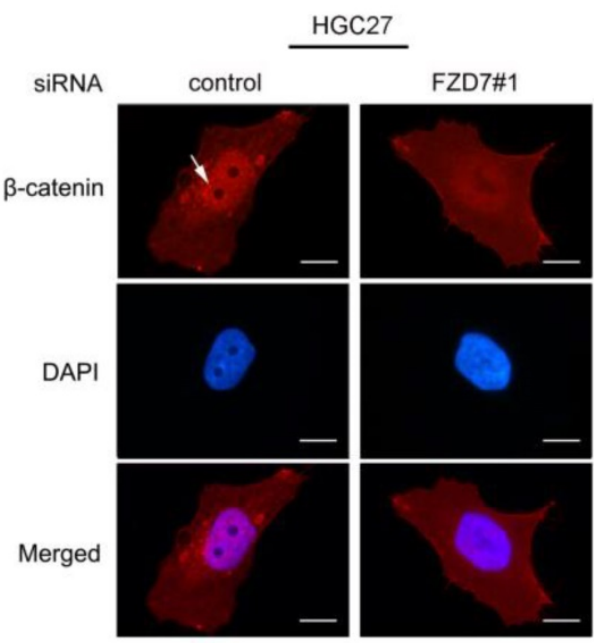
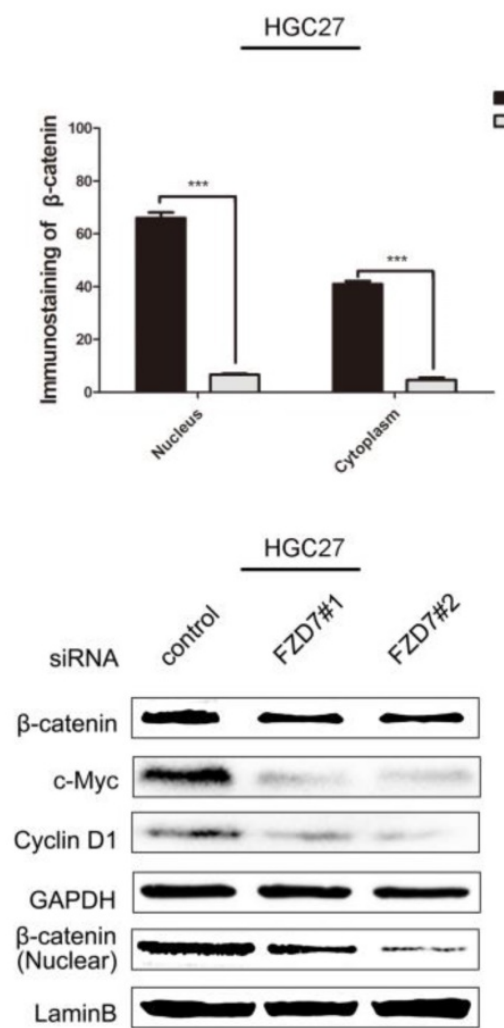

Fig.6 Knockdown of FZD7 inhibited Wnt/ $\beta$-catenin signaling pathway in gastric cancer cells. (A) Immunofluorescence assay showed that silencing of FZD7 decreased the accumulation of $\beta$-catenin in both nucleus and cytoplasm compared with their control cells. White arrows indicated $\beta$-catenin accumulation in nucleus. (scale bars:10 $1 \mathrm{~m}$ ). (B) Quantifications of $\beta$-catenin accumulation in nucleus and cytoplasm. (***indicates $P<0.001$, independent Student's $t$-test). (C) Western blot analysis showed that FZD7 depletion suppressed the expression of the canonical Wnt pathway target genes c-Myc and Cyclin DI and decreased $\beta$-catenin expression. GAPDH was used as a loading control. 
Table 2. Association of various factors with overall survival determined by COX regression.

\begin{tabular}{|c|c|c|c|c|c|c|}
\hline \multirow[t]{2}{*}{ Variable } & \multicolumn{3}{|c|}{ Univariate COX analysis } & \multicolumn{3}{|c|}{ Multivariate COX analysis } \\
\hline & HR & $95 \% \mathrm{CI}$ & $P$ & HR & $95 \% \mathrm{CI}$ & $P$ \\
\hline Sex (male vs. female) & 0.824 & $0.569-1.193$ & 0.305 & 0.957 & $0.648-1.412$ & 0.824 \\
\hline Age $(\leq 56$ vs. $>56)$ & 0.985 & $0.701-1.384$ & 0.931 & 1.021 & $0.717-1.455$ & 0.907 \\
\hline Histology type & 1.023 & $0.870-1.204$ & 0.782 & 1.011 & $0.848-1.205$ & 0.905 \\
\hline TNM stage (I-IIvs.III-IV) & 4.183 & $2.954-5.925$ & $<0.0001$ & 3.455 & $2.376-5.025$ & $<0.0001$ \\
\hline FZD7 expression (low expression vs. high expression) & 2.489 & $1.767-3.507$ & $<0.0001$ & 1.681 & $1.153-2.450$ & 0.007 \\
\hline
\end{tabular}

\section{Discussion}

In the past decades, intense research has demonstrated an essential role of Wnt/FZD7 signaling in regulating cell differentiation, proliferation and tissue homeostasis. Many cancers also display up-regulation of FZD7 which is associated with cancer cell proliferation and invasiveness, suggesting that FZD7 transmitting Wnt signaling is important for driving cancer growth. FZD7 mRNA expression has been found to be higher in late stage colorectal cancers and patients with higher FZD7 expression have unfavorable overall survival. Furthermore, knockdown of FZD7 expression dramatically attenuates invasion and metastatic capabilities of colon cancer cells [10]. Over-expression of FZD7 in AGS and BGC823 gastric cancer cells also increases cell proliferation [19]. Additionally, knockdown of FZD7 expression in triple negative breast cancer cells results in reduced cell proliferation and colony formation in vitro, and decreases tumor growth in NOD-SCID IL2rg female null mice [20]. In this study, we found that FZD7 was highly expressed in gastric cancers. FZD7 over-expression was associated with cancer metastasis, advanced clinical stages and poor patient prognosis. Down-regulation of FZD7 in gastric cancer cell lines caused impaired cell growth and invasion. These results are in line with the previous findings suggesting that FZD7 facilitates cancer development and progression.

EMT enables tumor cells to obtain migratory capacity, infiltrate surrounding tissue and metastasize to distant sites. Wnt signaling has been reported to promote EMT through up-regulating the transcription factors slug and twist [21]. Likewise, Frizzleds are known as critical factors for EMT processes. Wnt5a/FZD2-mediated noncanonical signaling drives EMT in liver, lung, colon, and breast cancer cell lines [22]. FZD4 ablation induces active $\beta 1$-integrin and E-cadherin expression, supporting that FZD4 regulates EMT and cell adhesion in prostate cancer cells [23]. As such, recent investigations show that down-regulation of FZD7 expression significantly inhibits cell invasion and migration, accompanied with decreased vimentin and snail, and increased E-cadherin in cervical and ovarian cancers [24, 25]. Wnt3/FZD7 signaling is up-regulated in hepatocell- ular carcinoma (HCC) and leads to a diminution of E-cadherin expression and an enhancement of K19 $[26,27]$. Over-expression of FZD7 could promote cell mobility, metastasis and EMT in esophageal cancer [28]. Our present study demonstrated that knockdown of FZD7 caused an increase of E-cadherin expression along with decreases of N-cadherin, fibronectin, vimentin, snail and MMP7. MMP7 is one of the most important downstream target genes of $\beta$-catenin, which disrupts E-cadherin/ $\beta$-catenin complex to up-regulate EMT transcription factors in prostate cancer $[29,30]$. MMP7 expression is upregulated in $\mathrm{H}$. pylori-infected gastric cancer cells and associated with EMT by cleavage of E-cadherin which leads to cancer cell migration and metastases [31]. Our work provided evidences that FZD7 could induce EMT and promote metastases in gastric cancer cells.

Among Frizzleds, Fzd7 is a predominant receptor to transmit Wnt signaling in regulation of human stem cell activity. Conditional deletion of FZD7 in adult intestinal epithelia results in stem cell loss and organoid death. Meanwhile, conditional deletion of FZD7 specifically in the Lgr5+ intestinal stem cells at crypts leads to impairment of the epithelium regeneration in transgenic mice [32]. FZD7 is significantly elevated in human embryonic stem cells and could be a novel embryonic stem cell-specific surface antigen due to its involvement in embryonic stem cell self-renewal and pluripotent state maintenance [33]. Cancer stem cells are a small subpopulation of cells with capabilities of self-renewal and tumorigenicity, and have key roles in treatment resistance, recurrence, and metastasis. Recently regulatory mechanisms that FZD7-dependent enhancement of Wnt signaling promotes normal mammary stem cell (MaSC) activity have been validated in breast cancer, implying that stem cells in normal and malignant tissues may share common molecular bases [18]. Moreover, FZD7 is found to be a potential biomarker of the stem/progenitor cells of Wilm's tumor $[12,34]$. We showed here that FZD7 was up-regulated in sphere-forming cells and knockdown of FZD7 in gastric cancer cells reduced the expressions of Nanog, Oct-4, ABCG2, CD24, CD44 and CD133. FZD7 down-regulation dramatically inhibited gastric cancer cell proliferation and growth. Taken together, our and other studies suggest that 
FZD7 might perform as a molecular driver for cancer stem cell activity.

FZD7 is involved in both canonical and non-canonical Wnt signaling activation. The canonical Wnt/ $\beta$-catenin pathway is triggered by a Wnt ligand binding to a FZD receptor, a Wnt co-receptor and low density lipoprotein receptor-related protein 5 (LRP5) or LRP6. When the Wnt co-receptor is Ror2, the non-canonical Wnt pathway is activated. The noncanonical Wnt signaling has two branches that include the Wnt/PCP and the Wnt/ $\mathrm{Ca}^{2+}$ pathways [9]. FZD7-dependent canonical Wnt signaling promotes breast cancer stem cell activity [18]. Wnt3/FZD7 signaling activates canonical Wnt/ $\beta$-catenin pathway and promotes EMT in non-transformed hepatic cells [27]. On the other hand, FZD7 activates JNK through non-canonical signaling to accelerate melanoma metastasis [35]. Activation of non-canonical Wnt/PCP pathway by FZD7 has been reported to promote ovarian cancer aggressiveness [25]. In this study, we observed a decrease of $\beta$-catenin in FZD7-silencing cells followed by down-regulation of the canonical Wnt pathway target genes c-Myc and Cyclin D1. Our findings indicated that FZD7 could mediate cancer stem cell self-renewal and EMT in gastric cancer via canonical Wnt signaling pathway. These results implied that the Wnt canonical pathway may contribute to tumorigenesis and metastasis, suggesting that FZD7 could be a potential therapeutic target for gastric cancer in the future.

\section{Abbreviations}

FZD7: frizzled 7; CSC: cancer stem cell; GC: gastric cancer; EMT: epithelial-mesenchymal transition; b-FGF: basic fibroblast growth factor; EGF: epidermal growth factor; IRS: immunoreactivity scoring system; IHC: immunohistochemistry; TMA: tissue microarrays; siRNA: small interfering RNAs; CCK-8: cell counting kit-8; MMP7: matrix metalloproteinase-7; qPCR: real-time quantitative polymerase chain reaction detecting system.

\section{Supplementary Material}

Supplementary figures and tables.

http://www.ijbs.com/v14p0280s1.pdf

\section{Acknowledgements}

This work was supported by grants from The National Natural Science Foundation of China (81072047 and 81302079), The Science and Technology Planning Project of Guangdong Province (2016A030303005, 2014A030304016, 2014A020212083, 2016A020215047 and 2017A020215016), The Science and Technology Foundation of Shenzhen (KQTD201
40630100658078) and "Three and three " Project of The First Affiliated Hospital of Sun Yat-Sen University.

\section{Competing Interests}

The authors have declared that no competing interest exists.

\section{References}

1. Ferlay J, Soerjomataram I, Dikshit R, Eser S, Mathers C, Rebelo M, et al. Cancer incidence and mortality worldwide: sources, methods and major patterns in GLOBOCAN 2012. International journal of cancer. 2015; 136: E359-86.

2. Zhou J, Ma X, Bi F, Liu M. Clinical significance of circulating tumor cells in gastric cancer patients. Oncotarget. 2017; 8: 25713-20.

3. Chaffer CL, Weinberg RA. A perspective on cancer cell metastasis. Science (New York, NY). 2011; 331: 1559-64.

4. Chaffer CL, San Juan BP, Lim E, Weinberg RA. EMT, cell plasticity and metastasis. Cancer metastasis reviews. 2016; 35: 645-54.

5. Lau EY, Ho NP, Lee TK. Cancer Stem Cells and Their Microenvironment: Biology and Therapeutic Implications. 2017; 2017: 3714190.

6. Zhan T, Rindtorff N, Boutros M. Wnt signaling in cancer. Oncogene. 2017; 36: 1461-73.

7. Liu N, Zang S, Liu Y, Wang Y, Li W, Liu Q, et al. FZD7 regulates BMSCs-mediated protection of CML cells. Oncotarget. 2016; 7: 6175-87.

8. Gurney A, Axelrod F, Bond CJ, Cain J, Chartier C, Donigan L, et al. Wnt pathway inhibition via the targeting of Frizzled receptors results in decreased growth and tumorigenicity of human tumors. Proceedings of the National Academy of Sciences of the United States of America. 2012; 109: 11717-22.

9. King TD, Zhang W, Suto MJ, Li Y. Frizzled7 as an emerging target for cancer therapy. Cellular signalling. 2012; 24: 846-51.

10. Ueno K, Hazama S, Mitomori S, Nishioka M, Suehiro Y, Hirata H, et al. Down-regulation of frizzled-7 expression decreases survival, invasion and metastatic capabilities of colon cancer cells. British journal of cancer. 2009; 101: 1374-81.

11. Kirikoshi H, Sekihara H, Katoh M. Up-regulation of Frizzled-7 (FZD7) in human gastric cancer. International journal of oncology. 2001; 19: 111-5.

12. Schmuck R, Warneke V, Behrens HM, Simon E, Weichert W, Rocken C. Genotypic and phenotypic characterization of side population of gastric cancer cell lines. The American journal of pathology. 2011; 178: 1792-804.

13. World Health Organization(WHO). Tumor of the stomach.In:Hamilton SR,Aaltonen LA,eds.World Health Organization classification of tumors.Tumors of the digestive system. Lyon:IARC Press. 2000: 37-68.

14. Japanese Gastric Cancer, Association. Japanese Classification of Gastric Carcinoma - 2nd English Edition. Gastric cancer : official journal of the International Gastric Cancer Association and the Japanese Gastric Cancer Association. 1998; 1: 10-24.

15. UICC. In:Sobin LH,Wittekind C,eds.TNM classification of malignant tumours,6th edn. New York:Wiley-Liss. 2002.

16. Anastas JN, Moon RT. WNT signalling pathways as therapeutic targets in cancer. Nature reviews Cancer. 2013; 13: 11-26.

17. Yin Y, Grabowska AM, Clarke PA, Whelband E, Robinson K, Argent RH, et al. Helicobacter pylori potentiates epithelial:mesenchymal transition in gastric cancer: links to soluble HB-EGF, gastrin and matrix metalloproteinase-7. Gut. 2010; 59: 1037-45.

18. Chakrabarti R, Wei Y, Hwang J, Hang X, Andres Blanco M, Choudhury A, et al. DeltaNp63 promotes stem cell activity in mammary gland development and basal-like breast cancer by enhancing Fzd7 expression and Wnt signalling. Nature cell biology. 2014; 16: 1004-15, 1-13.

19. Geng Y, Lu X, Wu X, Xue L, Wang X, Xu J. MicroRNA-27b suppresses Helicobacter pylori-induced gastric tumorigenesis through negatively regulating Frizzled7. Oncology reports. 2016; 35: 2441-50.

20. Yang L, Wu X, Wang Y, Zhang K, Wu J, Yuan YC, et al. FZD7 has a critical role in cell proliferation in triple negative breast cancer. Oncogene. 2011; 30: 4437-46.

21. DiMeo TA, Anderson K, Phadke P, Fan C, Perou CM, Naber S, et al. A novel lung metastasis signature links Wnt signaling with cancer cell self-renewal and epithelial-mesenchymal transition in basal-like breast cancer. Cancer research. 2009; 69: 5364-73.

22. Gujral TS, Chan M, Peshkin L, Sorger PK, Kirschner MW, MacBeath G. A noncanonical Frizzled2 pathway regulates epithelial-mesenchymal transition and metastasis. Cell. 2014; 159: 844-56.

23. Gupta S, Iljin K, Sara H, Mpindi JP, Mirtti T, Vainio P, et al. FZD4 as a mediator of ERG oncogene-induced WNT signaling and epithelial-to-mesenchymal transition in human prostate cancer cells. Cancer research. 2010; 70: 6735-45.

24. Deng B, Zhang S, Miao Y, Zhang Y, Wen F, Guo K. Down-regulation of Frizzled-7 expression inhibits migration, invasion, and epithelial-mesenchymal transition of cervical cancer cell lines. Medical oncology (Northwood, London, England). 2015; 32: 102. 
25. Asad M, Wong MK, Tan TZ, Choolani M, Low J, Mori S, et al. FZD7 drives in vitro aggressiveness in Stem-A subtype of ovarian cancer via regulation of non-canonical Wnt/PCP pathway. Cell death \& disease. 2014; 5: e1346.

26. Kim M, Lee HC, Tsedensodnom O, Hartley R, Lim YS, Yu E, et al. Functional interaction between Wnt3 and Frizzled-7 leads to activation of the Wnt/beta-catenin signaling pathway in hepatocellular carcinoma cells. Journal of hepatology. 2008; 48: 780-91.

27. Nambotin SB, Tomimaru Y, Merle P, Wands JR, Kim M. Functional consequences of WNT3/Frizzled7-mediated signaling in non-transformed hepatic cells. Oncogenesis. 2012; 1: e31.

28. Cao TT, Xiang D, Liu BL, Huang TX, Tan BB, Zeng CM, et al. FZD7 is a novel prognostic marker and promotes tumor metastasis via WNT and EMT signaling pathways in esophageal squamous cell carcinoma. Oncotarget. 2017; 8: 65957-68.

29. Zhang Q, Liu S, Parajuli KR, Zhang W, Zhang K, Mo Z, et al. Interleukin-17 promotes prostate cancer via MMP7-induced epithelial-to-mesenchymal transition. Oncogene. 2017; 36: 687-99.

30. Chen L, Li M, Li Q, Wang CJ, Xie SQ. DKK1 promotes hepatocellular carcinoma cell migration and invasion through beta-catenin/MMP7 signaling pathway. Molecular cancer. 2013; 12: 157.

31. Varro A, Kenny S, Hemers E, McCaig C, Przemeck S, Wang TC, et al Increased gastric expression of MMP-7 in hypergastrinemia and significance for epithelial-mesenchymal signaling. American journal of physiology Gastrointestinal and liver physiology. 2007; 292: G1133-40.

32. Flanagan DJ, Phesse TJ, Barker N, Schwab RH, Amin N, Malaterre J, et al. Frizzled7 functions as a Wnt receptor in intestinal epithelial Lgr5(+) stem cells. Stem cell reports. 2015; 4: 759-67.

33. Fernandez A, Huggins IJ, Perna L, Brafman D, Lu D, Yao S, et al. The WNT receptor FZD7 is required for maintenance of the pluripotent state in human embryonic stem cells. Proceedings of the National Academy of Sciences of the United States of America. 2014; 111: 1409-14.

34. Pode-Shakked N, Harari-Steinberg O, Haberman-Ziv Y, Rom-Gross E, Bahar $\mathrm{S}$, Omer D, et al. Resistance or sensitivity of Wilms' tumor to anti-FZD7 antibody highlights the Wnt pathway as a possible therapeutic target. Oncogene. 2011; 30: 1664-80

35. Tiwary S, Xu L. FRIZZLED7 Is Required for Tumor Inititation and Metastatic Growth of Melanoma Cells. PloS one. 2016; 11: e0147638. 